\title{
ATLANTE

\section{SUSTENTO METODOLOGICO PARA UNA PROPUESTA DE FORMACIÓN DOCENTE PARA LA EXPERIENCIA EDUCATIVA DE INGLÉS DE LA LICENCIATURA EN LENGUA INGLESA DE LA UNIVERSIDAD VERACRUZANA}

\author{
Copérnico Fernando Pereyra Centella ${ }^{1}$ \\ ORCID: 0000-0002-4641-7245. \\ Facultad de Idiomas. Universidad Veracruzana. México. \\ e-mail: cfpc73@yahoo.com.mx
}

Para citar este artículo puede utilizar el siguiente formato:

Copérnico Fernando Pereyra Centella: "Sustento metodológico para una propuesta de formación docente para la experiencia educativa de inglés de la licenciatura en lengua inglesa de la universidad veracruzana", Revista Atlante: Cuadernos de Educación y Desarrollo, ISSN: 1989-4155 (vol 13, № 7 octubre-diciembre 2021, pp. 196-211). En línea:

https://doi.org/10.51896/atlante/GCES6475

\section{RESUMEN}

En este artículo se describe el sustento metodológico para el diseño de una propuesta de formación docente que impacte de manera positiva en la Experiencia Educativa de Inglés de la Licenciatura en Lengua Inglesa de la Universidad Veracruzana. Se destaca el papel de la formación por parte de los profesores, ya que la universidad contrata a sus maestros por un perfil profesional, pero no por un perfil docente; por lo que la capacitación continua es necesaria. Para el sustento de esta propuesta, se realizó un estudio de índole descriptivo con un enfoque cuantitativo. Se aplicaron tres cuestionarios a docentes, estudiantes y la parte administrativa, respectivamente. El análisis de la información recopilada confirma la necesidad de la formación para el mejor desarrollo del proceso de enseñanza de la Experiencia Educativa de inglés y también brinda los elementos necesarios para generar una propuesta.

Palabras clave: propuesta de formación docente, Experiencia Educativa, Universidad Veracruzana.

\section{METHODOLOGICAL SUPPORT FOR A PROPOSAL OF TEACHER TRAINING FOR THE EDUCATIONAL EXPERIENCE OF ENGLISH OF THE ENGLISH LANGUAGE DEGREE OF THE UNIVERSITY OF VERACRUZ}

\footnotetext{
${ }^{1}$ Cursó la Licenciatura en Lengua Inglesa, la Maestría en la Enseñanza del Inglés como Lengua Extranjera y el Doctorado en Educación. Es docente de la Licenciatura en Lengua Inglesa, modalidad presencial, y de la Licenciatura en Enseñanza del Inglés, Modalidad Virtual de la Facultad de Idiomas de la Universidad Veracruzana. e-mail: cfpc73@yahoo.com.mx; cpereyra@uv.mx
} 


\begin{abstract}
This article describes the methodological support for the design of a teacher training proposal that has a positive impact on the English Educational Experience of the English Language Degree of the University of Veracruz. The role of training is highlighted, since the university hires their teachers for a professional profile, but not for a teaching profile, so continues training is necessary. To support this proposal, a descriptive study was carried out with a quantitative approach. Three questionnaires were applied to teachers, students, and the administrative part, respectively. The analysis of the information collected confirms the need for training to have a better development of the English teaching process and provides the necessary elements to generate a proposal.
\end{abstract}

Keywords: teacher training proposal, Educational Experience, University of Veracruz.

\title{
INTRODUCCIÓN
}

La educación es un proceso complejo que impacta directamente en todo el quehacer social del individuo. Es un proceso que puede entenderse solamente a la luz de una serie de disciplinas que la abordan desde distintas aristas para tratar de entenderla en toda su extensión de una manera operativa y no sustantiva. Por ejemplo, la psicología educacional, pedagogía, didáctica, antropología, historia, derecho, etnografía, etc., para tener un entendimiento claro de lo que es la educación y de su devenir en cada momento de la historia desde que la educación se originó como tal. Y este proceso educativo se torna todavía más complejo porque siempre va a desarrollarse en un contexto social, histórico, político y cultural específico. En este sentido,

es obvio que el estudio de la educación es una parte importante del estudio de la historia de la sociedad, junto con el de su economía, su política, su demografía o sus modos de comunicación, por citar otros ámbitos sociales, asimismo relevantes, con los que guarda una estrecha relación. (Viñao, 2002, p. 248).

Asimismo, es un proceso social que ha buscado y busca responder preguntas tales como: ¿A quién educamos?, ¿Por qué educar?, ¿Cómo educar?, ¿Qué hay que enseñar?, ¿Cómo lograr que los estudiantes aprendan?, ¿Qué deben aprender los estudiantes? Aunque todas estas preguntas no son nuevas, pues ya se las planteaban en su momento los Sofistas, Sócrates, Aristóteles, Comenio, Tolstoi, la Salle, Freire, etc., en los últimos 25 años han cobrado mucho auge en el ámbito educativo. La responsabilidad mayor para dar respuesta a estas y otras interrogantes ha recaído mayormente en la universidad pública, debido a la misión social que tiene como formadora de ciudadanos social y democráticamente responsables y como gestora de cambios sociales, políticos, económicos y culturales. Cabe mencionar que uno de los mecanismos a través de los cuales la universidad pública está tratando 
de dar respuesta a estas preguntas, y de justificar su razón de ser ante la sociedad, es por medio de la formación docente de sus académicos. Es así como la intención de este trabajo es mostrar la descripción del sustento metodológico que siente las bases para el diseño de una propuesta de formación docente para la Experiencia Educativa de Inglés de la Licenciatura en Lengua Inglesa de la Universidad Veracruzana.

\section{Problematización}

La formación docente se presenta como una necesidad insoslayable entre los académicos, ya que la disciplina por su propia naturaleza no les brinda las herramientas pedagógicas y didácticas indispensables para ser buenos maestros. Es decir, la universidad los contrata por su perfil profesional, mas no por un perfil docente. De ahí surge la necesidad de contar con una formación docente acorde a las necesidades de cada programa educativo, de los maestros y estudiantes y que contribuya de manera positiva al perfil de egreso. En este sentido, el Plan de Trabajo 2015-2019 de la Facultad de Idiomas, que a su vez se fundamenta en el Programa de Trabajo Estratégico 2013-2017 de la Universidad Veracruzana, y que se basa en las propuestas de documentos y acuerdos internacionales como la Declaración de la Sorbona (1998), la Declaración de Bolonia (1999), la Declaración de Praga (2001), Proyecto Tunin en Europa (2001-2003) y Proyecto Tunin para América Latina (2004-2008 y 2011-2013), contempla a una planta académica de calidad en la que subyace la formación docente.

En este sentido, la Universidad Veracruzana en su Programa de Trabajo Estratégico 2013-2017 (pp. 17, 18) en su apartado PTE 2013-2017 2) Planta Académica con Calidad busca impulsar una formación y una actualización permanentes de los académicos que promuevan las competencias pedagógicas, didácticas y disciplinares, y que les posibilite participar en tutorías y en el manejo de las TIC para mejorar la calidad educativa. Por su parte el Plan de Trabajo de la Facultad de Idiomas (PlaDEAFI) en su apartado PLaDEAFI 2015 - 2019 Fortalecimiento de la Planta Académica,

Subprograma 11. Aseguramiento de una planta académica con calidad dice

que es necesario generar acciones que lleven a la certificación de las competencias profesionales y docentes del personal académico por parte de organismos nacionales e internacionales. Resulta sumamente importante para el aseguramiento de una planta académica de calidad, el promover permanentemente el mejoramiento del perfil académico integral. En el desempeño académico y la profesionalización docente, también se debe considerar su participación activa en el trabajo colegiado, el cual se constituye como una estrategia de consulta, reflexión, análisis y concertación entre los profesores de la entidad, ... enriqueciendo así el quehacer docente. Esto tiene como objetivo, entre otros, fortalecer las competencias profesionales y docentes. Una forma de lograr este objetivo es a través de la difusión de cursos de formación 
y actualización académica y consolidar las competencias académicas en términos de metodología de la enseñanza del idioma como lengua extranjera y mejoramiento del nivel de lengua. (Facultad de Idiomas, 2015, pp. 17, 18).

El imperativo para la formación docente también obedece a evaluaciones internas y externas a las que están sujetas las Instituciones de Educación Superior, y la Universidad Veracruzana no está exenta de ello, por organismos como los Comités Interinstitucionales de Evaluación de la Educación Superior (CIEES), el Consejo para la Acreditación de la Educación Superior (COPAES), la Asociación Nacional de Universidades e Instituciones de Educación Superior (ANUIES), la Comisión Nacional de Evaluación (CONAEVA) y el Sistema Institucional de Evaluación y Acreditación (SIEVAC). Cabe señalar que la evaluación es una tendencia mundial en la escuela pública. Dicha evaluación ha sido una exigencia no sólo por parte del sector político, sino también de los diferentes sectores de la sociedad. Es una exigencia válida, pues la universidad debe justificar su razón de ser, su permanencia y sus resultados. Esto es saludable porque finalmente la universidad pública se debe a la sociedad y el compromiso que tiene para formar a los ciudadanos es enorme.

Pero también hay una necesidad de una formación docente que no obedezca solamente a un plan de trabajo institucional o que sea el resultado de evaluaciones internas y externas para obtener mayores recursos económicos, sino que también sea una formación docente que coadyuve a la concreción del perfil de egreso de cada programa educativo y acorde al modelo educativo de la universidad, que en el caso de la UV es integral y flexible (surgido en 1999). Es un modelo que busca dar respuesta a los retos y demandas de los contextos sociales, culturales, políticos y económicos de nuestra época, a través de una educación integral, integra e integrada, con un enfoque por competencias y basada en valores que permitan formar ciudadanos competentes en mercado y democracia para que sus egresados puedan insertarse de manera óptima en la sociedad. Pero también se pretende que esta propuesta de formación docente tome en consideración los dos modelos pedagógicos que subyacen al Modelo Educativo Integral y Flexible (MEIF). Dichos modelos son el desarrollista y constructivista. En el modelo desarrollista el docente es un facilitador de experiencias que guía al estudiante para que construya sus propios contenidos de aprendizaje, toma en cuenta las características individuales del estudiante, procura una adecuación curricular, se trabaja por procesos y promueve un conocimiento complejo. Por su parte, el modelo constructivista le permite al estudiante construir conocimiento nuevo a partir del que ya tiene, considera al estudiante como un ser único, pero que pertenece a un contexto social determinado que impacta en él. Este modelo pedagógico reconoce el papel que la familia, la comunidad, el contexto y la escuela juegan en la construcción personal del estudiante. En este modelo los docentes debemos contestar a tres interrogantes principales: ¿Qué tipo de sujeto quiero formar?, ¿Qué tipo de escuela se requiere para formar a este sujeto? y ¿Para qué tipo de sociedad voy a formar a este sujeto? (Torres de Torres, 2016). 


\section{Programa de Formación de Académicos (ProFA)}

El ProFA es una estructura que tiene como objetivo principal ofrecer al personal académico de la Universidad Veracruzana una diversidad de cursos, talleres, seminarios y diplomados encaminados al fortalecimiento disciplinar y pedagógico de la planta docente.

Este proceso de formación, que se ofrece a través del Departamento de Formación Académica, encuentra su sustento legal en la cláusula 128 del Contrato Colectivo de Trabajo del personal académico, que a la letra dice que

La Universidad Veracruzana está obligada a proporcionar capacitación y adiestramiento, técnico y profesional, a todos sus trabajadores académicos, en términos de lo previsto en el artículo 132 fracción XV del Capítulo Tercero Bis, Título Cuarto de la Ley Federal del Trabajo. (2014, p. 63).

El Programa de Formación de Académicos también responde a las normas pedagógicas y políticas educativas emanadas del Modelo Educativo Integral y Flexible (MEIF) de la Universidad Veracruzana y de programas institucionales como el Proyecto Aula (2009-2012) e Institucionalización del proceso permanente de Innovación Educativa (2013) y el Programa de Trabajo Estratégico 2014-2017 en su eje I. Innovación académica con calidad, Programa 2. Planta académica con calidad.

Este programa busca desarrollar las siguientes competencias: Comunicación, Autoaprendizaje, Orientación, Diagnóstico, Gestión, Planeación, Organización, Ejecución, Intervención y Evaluación. Todo esto tiene como fin último contribuir al siguiente perfil docente:

Profesional con saberes pedagógicos, disciplinarios y socioculturales que gestiona el aprendizaje y propicia la formación integral de los académicos de la Universidad Veracruzana con características y perfiles profesionales diversos, mediante el diseño y operación de ambientes de aprendizaje presenciales, distribuidos y virtuales, vinculados a comunidades educativas, desarrollando procesos permanentes de comunicación y de investigación-aprendizaje-acción sobre la práctica docente, en el marco del Modelo Educativo institucional, a partir del ejercicio de cinco competencias y sus respectivos atributos: Comunicación, Planeación, Autoaprendizaje, Investigación y Evaluación.

(Dirección General de Desarrollo Académico e Innovación Educativa, 2014, p. 4) 


\section{Marco Teórico}

Todo proceso educativo se desarrolla siempre dentro de un marco social, ya sea como proceso de reproducción, de represión o de transformación. Y dentro de toda sociedad encontramos un conjunto de ideas, creencias y formas de comportamiento llamado cultura. Una cultura que determina prácticamente todo el quehacer social del hombre: su forma de ser, de pensar, de actuar, de relacionarse, de generar conocimiento. En este sentido, Abbagnano y Visalberghi señalan que "cultura es el conjunto, más o menos organizado y coherente, de los modos de vida de un grupo humano" (2000, p. 11). Asimismo, toda cultura es transmitida de generación en generación a través del proceso educativo que tiene lugar en la escuela. Una institución conformada por diversos agentes tales como los docentes, los estudiantes, las autoridades escolares y, en años más recientes, la familia, y cuyo objetivo es el de educar o enseñar a las generaciones presentes y futuras. Gimeno y Pérez definen a la escuela y su misión de una manera muy clara cuando dicen que la escuela es una "una institución configurada para desarrollar el proceso de socialización de las nuevas generaciones y garantizar la reproducción social y cultural como requisito para la supervivencia de la misma sociedad" (2005, p. 18).

\subsection{El profesor efectivo}

En este momento histórico, social y cultural de la educación, lo que se busca es contar con docentes efectivos (o competentes como dirían otros) que entiendan, comprendan y sean capaces de desarrollar un proceso de enseñanza y aprendizaje basado en un enfoque o paradigma por competencias. Esto es el resultado de una dinámica mundial producto no solamente de evaluaciones realizadas por organismos nacionales e internacionales como CIEES, COPAES, OCDE, sino también de la presión ejercida por el Banco Mundial (BM) y el Fondo Monetario Internacional (FMI). Organismos estos que han provocado que los procesos educativos se centren en la enseñanza para que la escuela prepare a los estudiantes para el trabajo a través del desarrollo de competencias, logrando la calidad educativa. Esto nos ha llevado a una visión pragmática de la educación donde ésta termina siendo un proceso individual, progresista, desarrollista, tecnológico, generador de riquezas y apolítico, donde quedan de lado las dimensiones pedagógicas y éticas de la educación. Por otra parte, tenemos una visión humanista de la educación donde ésta se concibe como integradora, incluyente, justa, libertaria, promotora de la paz. Esta visión está relacionada con la forma como la Organización de las Naciones Unidas para la Educación, la Ciencia y la Cultura (UNESCO) concibe a la educación, pero aun la UNESCO termina promoviendo la competitividad entre los países.

A fin de cuentas, lo que se busca es contar con docentes efectivos que encajen en un modelo educativo basado en competencias, que cuenten con las herramientas pedagógicas necesarias para impactar de manera positiva en los estudiantes para ayudarlos a desarrollar las competencias deseadas para el mercado laboral. Con relación a este punto, Dunkin afirma que la

efectividad docente es una cuestión referida a la capacidad del mismo para lograr los efectos deseados sobre los estudiantes. Además, define 
la competencia docente como el conocimiento y las habilidades necesarias, y del desempeño docente como la forma en que este se conduce durante el proceso de enseñar. (1997 citado en Hunt, 2009, p. $5)$.

Hunt profundiza en este sentido y dice que el término efectividad docente se refiere a las características, competencias y comportamientos de los docentes y que todo esto, en su conjunto, les brinda a los estudiantes las herramientas necesarias para alcanzar los objetivos propuestos, para ser personas críticas, para trabajar cooperativa y colaborativamente. Esto cobra relevancia porque es un indicador que nos dice, como docentes, hacia donde tenemos que dirigir nuestros esfuerzos para consolidar ciudadanos competentes en mercado y democracia.

\subsection{El maestro universitario y su formación docente}

El maestro universitario es un profesional de su disciplina, con un amplio conocimiento de la misma. Sin embargo, la disciplina por su propia naturaleza no lo forma para ser un buen docente. De tal manera que es responsabilidad del profesor formarse como docente de manera permanente. Desafortunadamente, no siempre resulta así, como dice Imbernón, el docente

se forma básicamente en el contenido científico de la materia que enseña y sobre la que investiga. Pero, por otra parte, resulta muy escaso, cuando no nulo, el interés del profesorado respecto a la formación necesaria para transmitir y compartir ese conocimiento con su alumnado. (2000, p. 41).

Esto es de suma importancia para contar con una definición operativa del profesor y proveer una formación docente de acuerdo con las necesidades de las Instituciones de Educación Superior (IES) y en concordancia con las necesidades y requerimientos locales, nacionales e internacionales. Por ejemplo, el Programa de Trabajo Estratégico 2013-2017: Tradición e Innovación de la Dra. Sara Deifilia Ladrón de Guevara González busca que todos los docentes se capaciten de manera continua a través de cursos pedagógicos y disciplinares; así como en la labor tutorial y el uso y promoción de las TIC.

\subsection{Formación Docente Permanente}

Debido a que la disciplina no faculta al profesor universitario para ser un buen docente, ya que éste no cuenta con formación inicial alguna, es menester que el profesor se capacite de manera permanente para ser un profesor eficiente y competente en docencia. En este sentido, Perrenoud define la competencia docente como "la que formula las cualidades individuales, de carácter ético, académico, profesional y social que debe reunir el docente" (2000, p. 37). Perrenoud (2005) también describe una serie de competencias, basadas en el referencial de competencias adoptado en Ginebra en 1996, que 
deberían formar parte de la formación continua del docente: organizar y animar situaciones de aprendizaje, gestionar la progresión de los aprendizajes, elaborar y hacer evolucionar dispositivos de diferenciación, trabajar en equipo, utilizar las nuevas tecnologías, afrontar los deberes y los dilemas éticos de la profesión, organizar la propia formación continua, etc.

Esto es imperativo para formar profesores que a través del saber-conocer, saber-hacer, saberser y saber-estar en la dimensión docente incidan de manera directa en el aprendizaje significativo de los estudiantes. Por su parte, Medina Rivilla dice que la formación docente es

la preparación y emancipación profesional del docente para elaborar crítica, reflexiva y eficazmente un estilo de enseñanza que promueva un aprendizaje significativo en los alumnos y logre un pensamiento-acción innovador, trabajando en equipo con los colegas para desarrollar un proyecto educativo común. (1998, p. 87).

Sobre este tenor de la formación docente y su impacto en el proceso de enseñanza y aprendizaje, Wilson, Shulman y Richert ofrecen siete categorías como constitutivas del conocimiento base de un formador o docente:

Conocimiento pedagógico general. Alude a las teorías del aprendizaje y de la instrucción, principios y técnicas de gestión del aula y programa formativo.

$>$ Conocimiento de los alumnos. Se refiere a la concreción de los principios del aprendizaje y del desarrollo, que el formador aplica sobre sujetos de la formación concretos. Incluye, además, el conocimiento de las actitudes hacia la temática, intereses y motivaciones.

Conocimiento del contexto. Hace referencia a una combinación de informaciones diversas sobre el entorno físico, las limitaciones oficiales, etc.

> Conocimiento del currículo. Está relacionado con los materiales y programas que se emplean para la formación en distintas instituciones.

$>$ Conocimiento de los fines educativos. Se refiere al conocimiento de los valores y de sus bases filosóficas.

Conocimiento de la materia. Se refiere al dominio de los contenidos científicos, técnicos, artísticos o filosóficos del área en cuestión, así como a las diversas formas posibles de organizar ese contenido. 
Conocimiento didáctico del contenido. Hace referencia al conocimiento específico que los formadores desarrollan acerca de cómo hacer comprensible la temática a los participantes de la formación. (1987 citado en Imbernón [Coord.], 2016, p. 19).

\section{Marco Metodológico}

\subsection{Objetivo general}

Describir el sustento metodológico que siente las bases para el diseño de una propuesta de formación docente para la Experiencia Educativa de Inglés de la Licenciatura en Lengua Inglesa de la Universidad Veracruzana, que impacte de manera positiva en la impartición de esta Experiencia Educativa y en la forma de evaluar los contenidos de ésta.

\subsection{Tipo de estudio}

Es un estudio a pequeña escala de índole descriptivo y el enfoque es cuantitativo. La intención aquí fue realizar un análisis sistemático de los puntos de vista y acciones de los sujetos que fungieron como muestra de este trabajo. Amén de esto, el estudio es de corte cuantitativo porque se analizó una parte delimitada y concreta del quehacer docente de una sola experiencia educativa de un programa y se buscó ser lo más objetivo posible.

\subsection{Contexto}

Este estudio se llevó a cabo en la Facultad de Idiomas de la Universidad Veracruzana, localizada en Francisco Moreno esq. Ezequiel Alatriste S/N, C.P. 91020, en la colonia Francisco Ferrer Guardia, en la ciudad de Xalapa, Veracruz. De manera particular, se tomó en cuenta a la Licenciatura en Lengua Inglesa. Esta licenciatura consta de 318 créditos, divididos en siete periodos con la finalidad de que el estudiante estándar egrese en tres años máximo. Sin embargo, la flexibilidad del programa permite que algunos estudiantes la cursen hasta en once periodos.

\subsection{Participantes}

Se aplicó un cuestionario a 7 de los 10 docentes que imparten la EE de Inglés en la Licenciatura en Lengua Inglesa. Cabe mencionar que algunos de ellos imparten más de un nivel de inglés o más de una sección de un mismo nivel. Cinco de estos docentes cuentan con grado de Maestría, uno con grado de Doctorado y uno tiene solamente grado de licenciatura. Seis de ellos toman por lo menos un curso de formación docente por año escolar. Así mismo, se aplicó otro cuestionario a 35 estudiantes, cinco por docente. Finalmente, la Directora de Facultad de Idiomas contestó un cuestionario diseñado especialmente para la parte administrativa. 


\subsection{Instrumento de recolección de datos}

El instrumento que se utilizó fue el cuestionario. Se aplicaron tres en total: el de los docentes, el de los estudiantes y el de la parte administrativa. Los tres cuestionarios tienen el propósito de obtener información sobre la formación y práctica docente.

El cuestionario de los docentes (Auto Evaluación Docente) está dividido en cuatro categorías: 1) Características de la carrera docente, con 10 reactivos; 2) Distribución del tiempo del trabajo académico, con 6 reactivos; 3) Características de la planificación del currículo, con 9 reactivos; y 4) Características de la evaluación, con 4 reactivos.

El cuestionario de los estudiantes (Evaluación del Desempeño Docente) consta de 15 preguntas que tienen que ver con la práctica docente del maestro en el salón de clases. Este cuestionario busca obtener información relacionada, por ejemplo, con el programa de la EE de inglés, las actividades, la transversalidad, dominio de los contenidos, la evaluación, recursos didácticos.

El cuestionario que se le aplicó a la directora de la Facultad de Idiomas (Directivos) consta de 13 preguntas. El objetivo de este cuestionario es obtener información, por ejemplo, sobre la importancia e impacto de la formación docente, la carga académica, el trabajo colegiado, la evaluación de la EE de inglés, promoción de la formación en docencia, la transversalidad.

Con respecto a los cinco estudiantes que se eligieron por docente, aunque algunos maestros imparten más de un nivel de inglés o más de una sección, no fue necesario aplicar el cuestionario a cinco estudiantes de cada sección, pues el cuestionario de los docentes busca obtener información sobre lo que el maestro hace y la forma como se comporta en la clase de inglés y no en un nivel o sección en particular.

\subsection{Recursos de evidencia}

Con la intención de aumentar la validez en los resultados (Yin, 1984), en este estudio se utilizó:

1. Análisis documental. Se analizó el diseño y la estructura del Programa de Formación de Académicos, que tiene como referente la metodología presentada en la Guía para el Diseño de Proyectos Curriculares con el Enfoque de Competencias, UV.

2. Análisis de archivos. Se revisaron los informes anuales de trabajo del año 2011 a la fecha.

3. Encuesta estructurada. Se aplicó a siete docentes, a 35 estudiantes y a la Directora de la Facultad de Idiomas para conocer sobre la formación y la práctica docente.

\subsection{Análisis de resultados}

En este apartado se describen solamente los resultados que se consideran como fortalezas de la formación y práctica docente y aquellas áreas de oportunidad para una propuesta de formación docente.

\subsubsection{Cuestionario de los docentes}


De la categoría Características de la carrera docente, el $85.7 \%$ de la muestra toma cursos de actualización con miras a obtener una promoción profesional en la Facultad de Idiomas, y también lo hacen con la intención de tener un mejor desempeño docente. Así mismo, el 71.4\% considera que su desempeño docente es idóneo y cumple con los criterios de calidad establecidos por la universidad y participa activamente de los cursos de actualización promovidos por la universidad. En cuanto a las áreas de oportunidad, solamente el $57.1 \%$ considera como prioritaria la formación en docencia para el mejoramiento de la práctica docente; mientras que sólo el $14.2 \%$ considera que los temas desarrollados en los cursos de actualización responden a las exigencias educativas actuales.

Con respecto a la categoría Distribución del tiempo del trabajo académico, el $71.4 \%$ de los maestros encuestados que imparten la EE de inglés forman parte de un Cuerpo Académico en la Facultad de Idiomas. Con respecto a la carga académica, sólo el $28.5 \%$ de los encuestados dicen que sí se difunden las políticas para la distribución de esta.

Con relación a la categoría Características de la planificación del currículo, el 71.4\% considera que su formación disciplinar le permite contribuir a lograr los perfiles de egreso de sus estudiantes; utilizan también recursos didácticos efectivos y útiles que responden a las exigencias educativas actuales; asimismo, le indican a los estudiantes los recursos bibliográficos, didácticos y tecnológicos a emplearse para lograr un mejor aprendizaje en la EE de Inglés; estos docentes utilizan materiales elaborados por ellos para ayudar a la construcción del aprendizaje. Dentro de las áreas de oportunidad, tenemos que sólo el $42.8 \%$ de los encuestados vincula de una manera práctica los contenidos de la Experiencia Educativa (EE) de inglés con otras EE.

En cuanto a la categoría Características de la evaluación, el $85.7 \%$ considera que el sistema de evaluación de los aprendizajes debe constar de diversos tipos de evaluación y de varias técnicas e instrumentos de evaluación. Dentro de las áreas de oportunidad, solamente el $14.2 \%$ de los docentes participantes consideran que la evaluación que se realiza (el examen estandarizado más el portafolio de evidencias) en la EE de inglés es apropiado para evaluar el aprendizaje de los estudiantes. Asimismo, sólo este porcentaje de docentes considera que la evaluación arriba descrita está en concordancia con el modelo educativo y pedagógico de la Universidad Veracruzana.

\subsubsection{Cuestionario de los estudiantes}

Con respecto a las fortalezas que los alumnos expresaron relacionadas con la evaluación del desempeño docente, tenemos lo siguiente:

$>$ El $71.4 \%$ de los encuestados afirmaron que el docente les proporcionó el programa de la Experiencia Educativa (EE) al inicio del curso.

$>$ El $65.7 \%$ de los estudiantes reconocen que el docente domina los contenidos de la asignatura de inglés de forma eficiente. Relacionado con este punto, aunque ligeramente por debajo de la media, un $45.7 \%$ opina que el docente siempre es claro en su exposición al impartir la clase. 
> De los estudiantes participantes, el $62.8 \%$ considera que la evaluación que se realiza (el examen estandarizado más el portafolio de evidencias) en las EE de inglés es apropiado para evaluar su aprendizaje. Este dato resulta interesante porque de los docentes que participaron en este estudio, sólo el $14.2 \%$ consideran que la evaluación contribuye a evaluar el aprendizaje de los estudiantes.

Dentro de las áreas de oportunidad, los resultados muestran lo siguiente:

> Un $48.5 \%$ de los estudiantes manifiestan que sólo de manera regular existe una relación entre el programa de la EE de inglés presentado por el docente y las actividades realizadas en el desarrollo de la asignatura.

$>$ El $42.8 \%$ de los encuestados manifiesta que el docente relaciona los contenidos estudiados con aspectos propios del perfil de egreso de la Licenciatura en Lengua Inglesa. Aunque éste fue el porcentaje mayor, es necesario trabajar en este rubro, ya que el resultado de la encuesta se encuentra por debajo de la media.

$>$ Con respecto a las formas más comunes con las cuales el profesor imparte la clase, ejercicios de gramática y vocabulario fue escogida un $85.7 \%$, mientras que ejercicios de las cuatro habilidades de la lengua fue seleccionada solamente un $42.8 \%$. Este resultado es preocupante en cierta medida porque da a entender que los docentes siguen impartiendo la EE de inglés de manera muy similar a como lo hacían en el plan de estudios anterior, Plan 90, donde el libro de texto estaba en caminado a preparar a los estudiantes para presentar exámenes internacionales de lengua. Sin embargo, el Plan MEIF 2008 se basa en un enfoque por competencias y se espera que el docente, actualmente considerado facilitador, guíe a los estudiantes para que logren desarrollar las competencias necesarias que van a necesitar en el mercado laboral una vez que egresen. Es por ello que el libro que se utiliza actualmente en la EE de inglés es el método LIFE, encaminado al desarrollo de competencias en las cuatro habilidades de la lengua. Dicho en otras palabras, no hay una relación entre el modelo educativo, el modelo pedagógico y el modelo didáctico.

$>$ El $62.8 \%$ de los estudiantes afirman que sólo de manera regular se han logrado vincular de una manera práctica los contenidos de la EE de inglés con otras experiencias educativas. Ese dato está totalmente relacionado con la respuesta de los docentes, ya que sólo el $42.8 \%$ de ellos lleva a cabo dicha vinculación.

> Sólo un $20 \%$ de los participantes respondieron que el maestro siempre utiliza recursos y materiales didácticos en el desarrollo de su clase. Esto dista de la respuesta de los docentes, ya que el $71.4 \%$ de ellos afirmaron utilizar estos tipos de recursos.

$>$ Con respecto a la evaluación que se realiza en la EE de inglés, los dos componentes que los estudiantes eligieron con mayor frecuencia son el portafolio de evidencias y el examen estandarizado, con un $77.1 \%$ y un $62.8 \%$, respectivamente. Aquí también debe trabajarse con mucho cuidado, pues hay otros tipos de evaluación que pueden realizarse con base en un enfoque por competencias. 


\subsubsection{Cuestionario de la parte administrativa}

Este cuestionario fue contestado por la directora de la Facultad de Idiomas de la Universidad Veracruzana. Sus respuestas respecto aquellas preguntas que contribuyen a la propuesta de formación docente son las siguientes:

\section{Dentro de las fortalezas}

$>$ Difunde las políticas para la distribución de la carga académica de sus maestros.

$>$ Promueve la participación de sus maestros en equipos multidisciplinarios de docentes que permitan establecer propuestas de innovación educativa.

> Promueve la participación de los docentes en Cuerpos Académicos.

$>$ Promueve la formación en docencia de sus profesores a través de cursos virtuales.

\section{Áreas de oportunidad}

$>$ Considera parcialmente que la formación docente del maestro influye en la forma en que los profesores imparten la clase.

> Considera parcialmente que el desempeño docente de sus maestros es idóneo y cumple con los criterios de calidad establecidos por la universidad. Esta opinión difiere un poco respecto a la de los docentes, ya que un $71.4 \%$ de ellos considera su desempeño como idóneo y de calidad de acuerdo a los criterios de la UV.

$>$ Considera parcialmente que la evaluación (el examen estandarizado más el portafolio de evidencias) en la EE de inglés es apropiada para evaluar el aprendizaje de los estudiantes. Aquí hay coincidencia con los docentes.

$>$ Promueve sólo de manera parcial, a través de la Academia de Inglés, la vinculación de una manera práctica de los contenidos de la EE de inglés con otras experiencias educativas.

\section{Componentes eje para la propuesta de formación docente}

Con base en los resultados de los cuestionarios, la propuesta de formación docente podrá encaminarse a la actualización docente para los profesores que imparten la Experiencia Educativa de inglés, en sus diversos niveles y secciones. Dicha propuesta de formación se enfocará en el abordaje de los contenidos de la Experiencia Educativa de inglés desde tres componentes eje: capacitación, evaluación y recursos didácticos.

\section{Alcances y limitaciones}

Dentro de los alcances, la propuesta que se genere podrá impactar de manera positiva en el corto tiempo debido a que el número de docentes que imparten la Experiencia Educativa de inglés no es grande. Esto permite que se tomen decisiones de manera más rápida en la Academia de Inglés para que 
se ponga en práctica dicha propuesta una vez que se diseñe. Asimismo, la propuesta de formación docente podrá ser un punto de partida para que las demás Academias hagan lo propio para generar sinergia en beneficio del docente y de los estudiantes.

En cuanto a las limitaciones, geográficamente el estudio se limita al contexto de la Licenciatura en Lengua Inglesa de la Facultad de Idiomas de la Universidad Veracruzana. Así mismo, la población de estudio se concentra solamente en 7 de 10 docentes que imparten la Experiencia Educativa de inglés en diversos niveles y secciones, a 35 estudiantes, cinco por profesor y a la Directora de la Facultad de Idiomas. Esto puede ser un impedimento para que los resultados se generalicen al resto de los docentes

y estudiantes de las diversas experiencias educativas que conforman el Programa de la Licenciatura en Lengua Inglesa.

\section{Recomendaciones}

No debemos olvidar que la universidad nos contrata por un perfil más lo que la universidad requiera de nosotros. Actualmente se necesita que el docente sea un profesionista competente que ayude a los estudiantes a desarrollar las competencias necesarias para que se inserten de manera óptima en el mercado laboral y logren la empleabilidad. En ese sentido, todos los docentes tenemos la obligación moral de capacitarnos de manera continua a través de los cursos de formación docente impartidos por las Instituciones de Educación Superior. Sin embargo, por el momento esta disposición es voluntaria y se apela a la buena disposición de los profesores para capacitarse. Considero que la Universidad Veracruzana debe generar los mecanismos precisos para que todos los docentes accedan a capacitarse, con la intención de desarrollarse disciplinar, pedagógica y didácticamente para mejorar la calidad de la educación.

\section{CONCLUSIÓN}

El docente universitario es un profesionista con un alto compromiso social, pues en sus manos recae la responsabilidad de formar a los ciudadanos de una nación. Es menester que el profesor se actualice y se forme de manera permanente para adquirir y desarrollar las competencias en docencia que lo lleven a ser un maestro efectivo. El docente universitario debe fungir como guía de los estudiantes y contar con las herramientas pedagógicas y didácticas para ayudar a los alumnos a desarrollar las competencias, conocimientos, habilidades y valores necesarios para que logren insertarse de manera exitosa en el campo laboral. Es así como el profesor debe entender y comprender que la formación docente no se la brinda la disciplina por su propia naturaleza y que debe buscar la forma de actualizarse, formarse y capacitarse para ser un buen maestro. Tal es el propósito del sustento metodológico para una propuesta de formación docente que aquí se ha presentado. Una propuesta encaminada a la actualización docente y que se compone de tres componentes ejes: capacitación, evaluación y recursos didácticos. 


\section{REFERENCIAS}

Abbagnano, N. y Visalberghi, A. (2000). Historia de la pedagogía. Fondo de Cultura Económica.

Contrato Colectivo de Trabajo del personal académico. Universidad Veracruzana. https://colaboracion.uv.mx/rept/files/2015/03/071/Contrato-Colectivo-FESAPAUV-2014-2016.pdf

Declaración de la Sorbona. (1998). http://eees.umh.es/contenidos/Documentos/DeclaracionSorbona.pdf

Declaración de Bolonia. (1999). http://eees.umh.es/contenidos/Documentos/DeclaracionBolonia.pdf

Declaración

de

Praga.

(2001).

http://ehea.info/media.ehea.info/file/2001 Praque/47/4/2001 Prague Communique Spanish 553 474.pdf

Dirección General de Desarrollo Académico e Innovación Educativa. Dirección de Fortalecimiento Académico. Programa de Formación de Académicos. Universidad Veracruzana. http://www.uv.mx/dgdaie/files/2014/05/descripcionprofa.pdf

Facultad de Idiomas. Licenciatura en Lengua Inglesa. Universidad Veracruzana. https://www.uv.mx/idiomas/principal/lengua-inglesa/

Facultad de Idiomas. Plan de Trabajo 2015-2019. Hacia un Escenario Deseable. Universidad Veracruzana. $\quad$ http://www.uv.mx/idiomas/files/2016/01/PlandetrabajoDireccionldiomas20152019.pdf

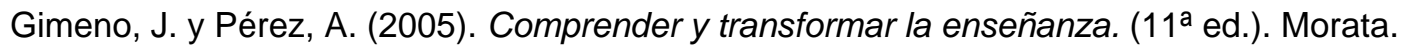

Hunt, B, C. (2009). Efectividad del desempeño docente. Una reseña de la literatura internacional y su relevancia para mejorar la educación en América Latina. San Marino.

Imbernón, F. (2000). Un nuevo profesorado para una nueva universidad. ¿Conciencia o presión? Red interuniversitaria de formación del profesorado, 28, 37-46. http://www.aufop.com/aufop/uploaded files/articulos/1223490004.pdf

Imbernón, F. (Coord.). (2016). Diseño, desarrollo y evaluación de los procesos de formación. Síntesis.

León Rivera, M. L., Carpio García, Z. S. y Cuadros Morales, I. (2011). Importancia de los enfoques Cuantitativos y Cualitativos de Investigación en Ciencias Sociales. Universidad del Valle de México.

Medina Rivilla, A. (1998). Organización de la formación y desarrollo profesional del docente universitario. $\checkmark$ Congreso Interuniversitario de Organización de Instituciones Educativas. Madrid.

Perrenoud, P. (2000). Diez Nuevas Competencias para Enseñar. Artmed.

Perrenoud, P. (2005). El trabajo sobre el habitus en la formación de maestros. Análisis de las prácticas y toma de conciencia. En L. Paquay, M. Altet, E. Charlier y P. Perrenoud. La formación profesional del maestro. Estrategias y competencias. Fondo de Cultura Económica.

Proyecto Tunin en Europa. (2001-2003).

https://www.unideusto.org/tuningeu/images/stories/documents/General Brochure Spanish versi on.pdf

Proyecto Tunin para América Latina. (2004-2008). http://tuning.unideusto.org/tuningal/ 
Proyecto Tunin para América Latina. (2011-2013). http://www.tuningal.org/

Torres de Torres, G. M. (2016). Modelos Pedagógicos. https://gingermariatorres.wordpress.com/modelos-pedagogicos/

Universidad Veracruzana. Programa de Trabajo Estratégico 2013-2017. Tradición e Innovación. https://www.uv.mx/programa-trabajo/Programa-de-Trabajo-Estrategico-version-para-pantalla.pdf

Viñao, A. (2002). La historia de la educación en el siglo XX. Una mirada desde España. Revista $\begin{array}{llllll}\text { Mexicana de Investigación } & \text { 223-256. }\end{array}$ http://www.redalyc.org/articulo.oa?id=14001503

Yin, R. (1984). Case study research Design and Methods. Sage Publications. 